\title{
MS38-P05 | RESOLUTION FUNCTION FOR 2D PIXEL DETECTORS
}

Chernyshov, Dmitry (SNBL at the ESRF, Grenoble, FRA); Dyadkin, Vadim (Swiss-Norwegian BeamLines at the European Synchrotron Radiation Facility, Grenoble, FRA)

Advent of low-noise high-energy pixel detectors open doors for fast diffraction experiments covering large Q-range in one shot, this is of a particular importance for powder diffraction and PDF measurements. Qualitative analysis of the powder diffraction data assumes a model for instrumental line broadening, most of the time it is parameterized with Cagliotti or alike functions [1]. Here we show that such a parameterization is not sufficient for large area detectors and propose a new resolution model accounting for beam divergency, pixel size, thickness of the detector' sensitive layer and geometrical parameters of the experiment. The model is derived analytically and compared with the models reported previously [2,3]; it can be easily implemented in the software for profile or PDF analysis. The analytical derivations are illustrated with calibration measurements at BM01 beamline (SNBL at the ESRF [4]) equipped with Pilatus2M detector.

[1] Gozzo, F., De Caro, L., Giannini, C., Guagliardi, A., Schmitt, B., \& Prodi, A. Journal of Applied Crystallography, 39(3), 347-357, (2006)

[2] Norby, P. Journal of Applied Crystallography, 30(1), 21-30, (1997)

[3] Hinrichsen B., Dinnebier R. E. and Jansen M. in "Powder Diffraction: Theory and Practice", edited by R. E. Dinnebier and S. J. L. Billinge, The Royal Society of Chemistry (2008)

[4] Dyadkin, V., Pattison, Ph., Dmitriev, V., Chernyshov, D. J. Synchrotron Rad., 23, 3, (2016) 